\title{
Brane world regularization of point particle classical self-energy
}

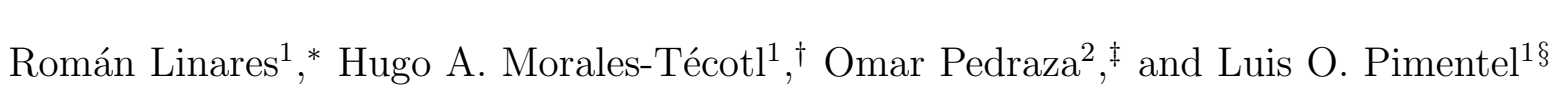 \\ 1 Departamento de Física, Universidad Autónoma Metropolitana Iztapalapa, \\ San Rafael Atlixco 186, C.P. 09340, México D.F., México, and \\ 2 Área Académica de Matemáticas y Física, \\ Universidad Autónoma del Estado de Hidalgo, \\ Carretera Pachuca-Tulancingo Km. 4.5, C P. 42184, Pachuca, México.
}

\begin{abstract}
Physical effects in brane worlds models emerge by the incorporation of field modes coming from extra dimensions with the usual four dimensional ones. Such effects can be tested with well established experiments to set bounds on the parameters of the brane models. In this work we extend a previous result which gave finite electromagnetic potentials and self energies for a source looking pointlike to an observer sitting in a 4D Minkowski subspace of a single brane of a RandallSundrum spacetime including compact dimensions, and along which the source stretches uniformly. We show that a scalar particle produces a nonsingular static potential, possess a finite self-energy and that technically its analysis is very similar to the electrostatic case. As for the latter, we use the deviations from the Coulomb potential to set bounds on the anti de Sitter radius of the brane model on the basis of two experiments, namely, one of the Cavendish type and other being the scattering of electrons by Helium atoms. We found these are less stringent than others previously obtained using the Lamb shift in Hydrogen.

PACS numbers: 11.25.Wx, 11.10Kk, 11.25.Mj,04.25.-g, 03.50.-z
\end{abstract}

*Electronic address: lirr@xanum.uam.mx

${ }^{\dagger}$ Electronic address: hugo@xanum.uam.mx

${ }^{\ddagger}$ Electronic address: omarp@uaeh.edu.mx

$\S$ Electronic address: lopr@xanum.uam.mx 


\section{INTRODUCTION}

Despite the extraordinarily rich accuracy with which the predictions of electrodynamics have been experimentally tested over the years (see for instance [1, 2] and references therein), efforts to place limits on deviations from its standard formulation continue nowadays. The nature of the experiments cover a big range of possibilities which include among others: a) Testing the power in the inverse-square law of Coulomb, b) Seeking a nonzero value for the rest mass of the photon and c) Considering more degrees of freedom, allowing mass for the photon while preserving explicit gauge invariance. It is worth to mention that all these experiments have probed length scales increasing dramatically over time.

Now, historically, once the Maxwell theory of electromagnetic fields was established, one of the main concerns in physics was the construction of a consistent description of electrodynamics and charged particles. The first serious proposals in this direction were developed by Lorentz [3] and Abraham [4]. These proposals and subsequent attempts based on classical electrodynamics, special relativity and the Lorentz force law led to the theory known as Classical Electron Theory (CET). Whereas in the Maxwell theory charges are considered as punctual which produce infinite Lorentz self-force and infinite electromagnetic self-energies associated with the singularities of the Liénard-Wiechert potential, in the CET charges are considered as extended objects that experience a volume-averaged Lorentz force. Parallel to the development of the CET, quantum mechanics was developed giving origin to one of the most spectacular theory we have in physics: Quantum Electrodynamics (QED). This theory is awesome due to the impressive range of electromagnetic phenomena it covers with spectacular precision. After the experimental achievements of QED, CET dropped from the list of contenders for a fundamental theory of electrodynamics interacting with matter. However despite the great success of QED there are still some features of the theory that could be waiting for a better explanation, for instance, its property of renormalizability. It turns out that QED is defined by a perturbative series that is renormalizable in each order, but it is most likely to be merely asymptotic in character rather than convergent [5], in such a way that the precision results are obtained only when computations are made to some order in the expansion series, but without any a priori prescription to stop the series at some order.

It is thus tempting to investigate theories that avoid singularities. These are not expected 
to solve the problem but at least they may contribute to a better understanding of the singularity issue.

In this context, recently, in a previous work, some of us found that a source lying on the single brane of a Randall-Sundrum spacetime including compact dimensions, and which looks pointlike to an observer sitting in usual 3D space, produces a static potential which is non singular at 3D point position. Moreover it matches Coulomb's potential outside a small neighborhood [6]. The presence of the compact dimensions in this setup serve to localize the gauge field on the brane [7-9]. The aim of this paper is to investigate further some consequences of the above property to set bounds to the AdS curvature radius $\epsilon$ using the experimental results from the Cavendish experiment for electromagnetism and the scattering of electrons by Helium atoms. For the sake of clarity, the simpler case of a scalar particle is first considered. Remarkably, the nonsingular character of the potential holds together with the finiteness of the selfenergy. Indeed, technically, the study of the potentials for both scalar and electromagnetic is very similar.

Our interest in this work is twofold, on one side it is interesting to explore how the old problem of divergences acquires a different character in light of the brane world models, at least classically, and, on the other hand, it is also interesting from the perspective of the brane-world scenarios [10 14], which have recently been matter of a copious research, mainly in high energy physics (see e.g. [15, 16], and references therein) and cosmology (see e.g. [17 19], and references therein). More recently, the possibility to obtain information from models with extra dimensions studying low energy physical phenomena has also been addressed. In particular we mention the ones that have been performed in the RSII- $p$ setup, such as the electric charge conservation [8], the Casimir effect between parallel plates [20, 21] and the Hydrogen Lamb shift [6].

The paper is organized as follows. In section II we briefly describe the RSII- $p$ setup, section III is dedicated to obtain the static potential for a scalar field whereas in section IV we do the same for the electric case. In section $\mathrm{VA}$ we set bounds to the AdS radius $\epsilon$ comparing our electrostatic results with the experimental values obtained in Cavendish like experiments of the Coulomb force. Section $\mathrm{VB}$ is dedicated to the same purpose but this time we use the experimental results of the scattering process of electrons by Helium atoms. Finally section VI is devoted to a brief discussion. 


\section{RANDALL-SUNDRUM II- $p$ SCENARIOS}

The Randall-Sundrum II- $p$ scenarios consist of a $(3+p)$-brane with $p$ compact dimensions and positive tension $\sigma$, embedded in a $(5+p)$ spacetime whose metrics are two patches of anti-de Sitter $\left(\mathrm{AdS}_{5+p}\right)$ having curvature radius $\epsilon$. The interest in these models comes from its property of localizing on the brane: scalar, gauge and gravity fields due to the gravity produced by the brane itself. This property is valid whenever there are $p$ extra compact dimensions [8, 9]. In the limiting case $p=0$, the model only localizes scalar and gravity fields. With this setup and appropriate fine-tuning between the brane tension $\sigma$ and the bulk cosmological constant $\Lambda$, which are related to $\epsilon$ as follows

$$
\sigma=\frac{2(3+p)}{8 \pi \epsilon G_{5+p}}, \quad \Lambda=-\frac{(3+p)(4+p)}{16 \pi \epsilon^{2} G_{5+p}}=-\frac{(4+p) \sigma}{4 \epsilon},
$$

there exists a solution to $(5+p)$ D Einstein equations with metric

$$
d s_{5+p}^{2}=e^{-2|y| / \epsilon}\left[\eta_{\mu \nu} d x^{\mu} d x^{\nu}-\sum_{i=1}^{p} R_{i}^{2} d \theta_{i}^{2}\right]-d y^{2} .
$$

Here $\eta_{\mu \nu}$ is the $4 \mathrm{D}$ Minkowski tensor, $\theta_{i} \in[0,2 \pi]$ are $p$ compact coordinates, $R_{i}$ are the sizes of compact dimensions, $G_{5+p}$ is the $(5+p)$ D Newton constant. Throughout the paper we will use the following notation for the $5+p$ coordinates $X^{M} \equiv\left(x^{\mu}, \theta_{i}, y\right)$, where $\mu=0,1,2,3$, and $i=1, \ldots p$.

In this work we consider two different $(5+p) D$ field theories on RSII- $p$ : a massless scalar field and electrodynamics. They will be subjected to a hybrid of the two well known consistent compactifications, namely Kaluza-Klein (KK) [22, 23] and warped [10]. These two differ among them on whether the compactified manifold is factorizable or not. The corresponding effective field theories in 4D Minkowski space-time will be given.

In regard to the KK compactification, it is well known toroidal dimensional reductions lead to consistent lower dimensional theories which nonetheless can be questioned in that they do not come with a mechanism to fix the moduli, or equivalently, the radii of the $p \mathrm{D}$ torus $T^{p}[24,25]$. Historically, a way out in such cases, has been to conform with the corresponding phenomenology at low enough energies and set a bound for the radii (e.g. the use of the classical value of the electron charge required a radius of the order Planck length in the original KK setting [23, 26]). We will adhere to this approach by considering a low energy approximation so that we truncate the massive KK modes of the compact dimensions 
but keep those corresponding to the noncompact dimension just meaning that we assume the energy scale of the former is much smaller than that of the latter. This is explicitly performed in the Green's function in $\Pi 1 \mathrm{~A}$ for the scalar field and in $\mathrm{IV}$ for the gauge field. As for the consistency of the Randall-Sundrum compactification it has been discussed in [27] (and references therein).

For completeness we only mention other mechanisms adopted in the literature to perform a generalized KK compactification. One of them is the so called Scherk-Schwarz compactification [28, 29] or flux compactifications [30 32]. In this mechanism the symmetries of the compactification manifold and/or the fields are used to produce an effective potential for stabilizing the size of the extra dimensions. There also exists a quantum proposal by Candelas and Weinberg [33] where the effective potential for the moduli fields is produced by the Casimir energy of matter fields or gravity. It remains open to study these possibilities for our present setup.

A remark regarding the stability of the scenario described by the metric (21) is here in order. Concerning the world volume of the $(3+p)$-brane, $M_{4} \otimes T^{p}$, it is clear that the space is stable since it is flat. On the other hand the stability of the space-time (2), without the $T^{p}$ structure, was studied long ago in [10, 34] for static perturbations of the metric and in [35] for general space-time dependent sources. The stability of other warped compactifications has also been addressed, for instance in [36-39] it was discussed the moduli stabilization of the RSI model whereas in [40] it was discussed for more general metrics.

Before ending this section is worth to mention that this setup has been considered in different low energy physics effects such as the electric charge conservation [8], the Casimir

effect between two conductor hyperplates $[20,21,41,42]$ and the Liennard-Wiechert potentials and Hydrogen Lamb shift [6] among others.

\section{STATIC POTENTIAL FOR A SCALAR FIELD}

In this section we compute the potential produced by a static source which is seen as punctual by an observer living on the usual 3D subspace of the $(3+p)$-brane. It stretches however along the $p$ compact dimensions thus forming a $p$-dimensional torus (11). 


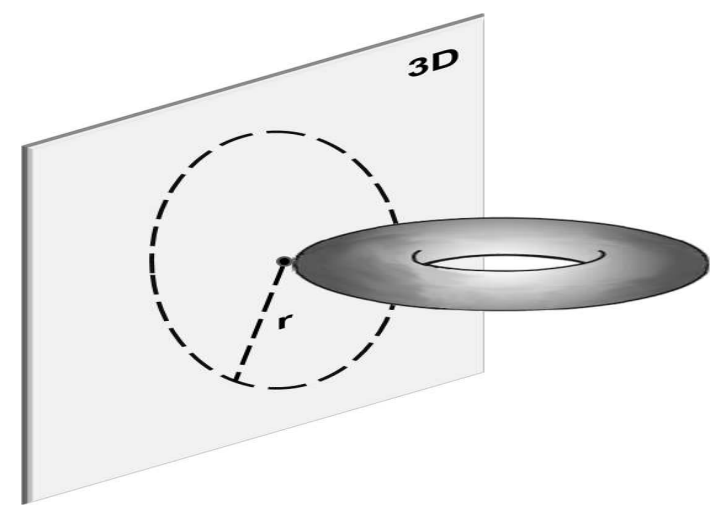

FIG. 1: Schematic view of the charge source for $p=2$. The source is effectively pointlike from the perspective of an observer sitting in the usual $3 \mathrm{~d}$ space.

\section{A. The Green's function}

Let us consider a massless scalar field $\Phi$ described by the action in $(5+p) \mathrm{D}$

$$
S=\int d^{4} x \prod_{i=1}^{p} R_{i} d \theta_{i} d y \sqrt{|g|}\left(\frac{1}{2} g^{M N} \partial_{M} \Phi \partial_{N} \Phi+\Phi J_{\text {scalar }}\right) .
$$

The equation of motion for the scalar field is

$$
\frac{1}{\sqrt{|g|}} \partial_{M}\left(\sqrt{|g|} g^{M N} \partial_{N} \Phi\right)=J_{\text {scalar }}
$$

where the source is given by

$$
J_{\text {scalar }}=\lambda^{(p)} \delta^{3}\left(\vec{x}-\vec{x}_{0}\right) \delta\left(y-y_{0}\right)
$$

Here $\lambda^{(p)}$ is a constant whose dimensions are $[$ charge $] /[\text { length }]^{p}$, explicitly: $\lambda^{(p)}=\frac{\lambda}{(2 \pi)^{p} R_{1} \cdots R_{p}}$, with $\lambda$ the total charge.

In the background (2), the equation of motion (44) becomes

$$
e^{2|y| / \epsilon}\left[\square \Phi-\sum_{i=1}^{p} \frac{1}{R_{i}^{2}} \partial_{\theta_{i}}^{2} \Phi\right]-\frac{1}{\sqrt{|g|}} \partial_{y}\left[\sqrt{|g|} \partial_{y} \Phi\right]=J_{\text {scalar }}
$$

where $\square$ stands for the flat 4D D'Alambertian. The corresponding Green's equation is

$$
e^{2|y| / \epsilon}\left[\square G-\sum_{i=1}^{p} \frac{1}{R_{i}^{2}} \partial_{\theta_{i}}^{2} G\right]-\frac{1}{\sqrt{|g|}} \partial_{y}\left[\sqrt{|g|} \partial_{y} G\right]=\frac{\delta\left(y-y^{\prime}\right) \delta^{p}\left(R_{i} \theta_{i}-R_{i} \theta_{i}^{\prime}\right) \delta^{4}\left(x-x^{\prime}\right)}{\sqrt{|g|}}
$$

where $G$ is the $(5+p)$ D Green's function. This can be expressed in terms of the eigenfunctions of the differential operators for the different coordinates. Assuming $\Psi\left(X^{M}\right) \equiv$ 
$e^{i k_{\mu} x^{\mu}} \prod_{i=1}^{p} \Theta_{i}\left(\theta_{i}\right) \psi(y)$, where the modes $\Theta_{n}$ and $\psi_{m}$ account for the $\theta$ and $y$ dependence respectively. These have been discussed previously (see for instance [7, 20]) and here we only give a summary. The differential equations governing the $p$ compact modes depending on $\theta_{i}$ are

$$
\left(\partial_{\theta_{i}}^{2}+m_{\theta_{i}}^{2} R_{i}^{2}\right) \Theta_{i}\left(\theta_{i}\right)=0, \quad i=1, \cdots, p
$$

whereas for the noncompact modes depending on $y$ one gets

$$
\left.\left(\partial_{y}^{2}-\frac{(4+p)}{\epsilon} \operatorname{sgn}(y) \partial_{y}\right)+m^{2} e^{2|y| / \epsilon}\right) \psi(y)=0 .
$$

The $(p+1)$ constants of separation, $m_{\theta_{i}}, m$, fulfill the following dispersion relation

$$
k^{2}=\sum_{i=1}^{p} m_{\theta_{i}}^{2}+m^{2} \equiv m_{p}^{2}+m^{2}
$$

To account for the compactness of the $p$ dimensions Eq. (8) is solved under the periodic boundary conditions

$$
\Theta_{n_{i}}\left(\theta_{i}\right)=\Theta_{n_{i}}\left(\theta_{i}+2 \pi\right)
$$

and the solutions turn out to be

$$
\Theta_{n_{i}}\left(\theta_{i}\right)=\frac{1}{\sqrt{2 \pi R_{i}}} e^{i n_{i} \theta_{i}}, \text { where } n_{i}=m_{\theta_{i}} R_{i} \in \mathbb{Z}
$$

To match the modes across the brane along the non-compact dimension, equation (9) is solved with the following boundary conditions

$$
\psi\left(y=0^{+}\right)=\psi\left(y=0^{-}\right) \quad \text { and } \quad \partial_{y} \psi\left(y=0^{+}\right)=\partial_{y} \psi\left(y=0^{-}\right) .
$$

In this case the solutions include a massless zero mode localized on the brane

$$
\psi_{0}(y)=\sqrt{\frac{2+p}{2 \epsilon}}
$$

which satisfies the normalization condition $2 \int_{0}^{\infty} d y e^{-(2+p)|y| / \epsilon} \psi_{0}^{2}(y)=1$, as well as massive modes given by

$$
\psi_{m}(y)=e^{\gamma y / \epsilon} \sqrt{\frac{m \epsilon}{2}}\left[a_{m} J_{\gamma}\left(m \epsilon e^{y / \epsilon}\right)+b_{m} N_{\gamma}\left(m \epsilon e^{y / \epsilon}\right)\right],
$$

where $J_{\gamma}$ and $N_{\gamma}$ are the Bessel and Neumann functions, respectively. In this expression

$$
\gamma \equiv \frac{4+p}{2}
$$


and the coefficients $a_{m}$ y $b_{m}$ are

$$
a_{m}=-\frac{A_{m}}{\sqrt{1+A_{m}^{2}}}, \quad b_{m}=\frac{1}{\sqrt{1+A_{m}^{2}}}, \quad A_{m}=\frac{N_{\gamma-1}(m \epsilon)}{J_{\gamma-1}(m \epsilon)} .
$$

Notice that in this case, the localization of the massive modes on the brane is better for increasing $p$, since the modes are modulated exponentially by a factor of $e^{-p|y| /(2 \epsilon)}$. The normalization condition for the massive modes is $\int_{-\infty}^{\infty} d y e^{-(p+2)|y| / \epsilon} \psi_{m}(y) \psi_{m^{\prime}}(y)=\delta\left(m-m^{\prime}\right)$.

With the eigenfunctions at hand it is straightforward to use them to write down the Green's function. It takes the form

$G\left(x, \theta_{i}, y ; x^{\prime}, \theta_{i}^{\prime}, y^{\prime}\right)=\prod_{i=1}^{p} \sum_{\{n\}} \frac{e^{i n_{i} \theta_{i}} e^{-i n_{i} \theta_{i}^{\prime}}}{2 \pi R_{i}} \int \frac{d^{4} k}{(2 \pi)^{4}} e^{i k_{\mu}\left(x^{\mu}-x^{\prime \mu}\right)}\left[\frac{\psi_{0}(y) \psi_{0}\left(y^{\prime}\right)}{k^{2}-m_{p}^{2}}+\int_{0}^{\infty} d m \frac{\psi_{m}(y) \psi_{m}\left(y^{\prime}\right)}{k^{2}-m^{2}-m_{p}^{2}}\right]$,

where $\{n\}$ denotes $\left\{n_{1}, n_{2}, \ldots, n_{p} \mid n_{1} \in \mathbb{Z}, \ldots, n_{p} \in \mathbb{Z}\right\}$.

At this point it is convenient to introduce an approximation that will allow us to obtain analytic expressions of the potential. We have massive modes from both compact dimensions (12) and the noncompact one (15). Since we are interested in the low energy regime we assume $m_{\theta_{i}} \ll m \ll \epsilon^{-1}$. Hence we will set $n_{1}=\cdots=n_{p}=0$, and, as for the noncompact modes (15) we use

$$
\psi_{m}(y) \approx-e^{\gamma y / \epsilon} \sqrt{\frac{m \epsilon}{2}} J_{\gamma}\left(m \epsilon e^{y / \epsilon}\right) .
$$

In such low energy regime and upon integrating over the $p$ compact extra dimensions, we end up with an effective 5D Green's function

$$
G_{5 D}\left(x, y ; x^{\prime}, y^{\prime}\right)=\int \frac{d^{4} k}{(2 \pi)^{4}} e^{i k_{\mu}\left(x^{\mu}-x^{\prime \mu}\right)}\left[\frac{\psi_{0}(y) \psi_{0}\left(y^{\prime}\right)}{k^{2}}+\int_{0}^{\infty} d m \frac{\psi_{m}(y) \psi_{m}\left(y^{\prime}\right)}{k^{2}-m^{2}}\right],
$$

where the massless mode is given by (14) and the massive modes by (19). Although we have only taken the zero modes of the compact extra dimensions, notice that their imprints remain in the 5D Green's function through (16) and (19).

\section{B. Static potential}

Now we are in position to compute the static potential. In this case the useful Green's function is

$$
\begin{aligned}
G\left(\vec{x}, y ; \overrightarrow{x^{\prime}}, y^{\prime}\right) & =\int_{-\infty}^{\infty} d t^{\prime} G\left(\vec{x}, t=0, y ; \overrightarrow{x^{\prime}}, t^{\prime}, y^{\prime}\right) \\
& =\frac{\psi_{0}(y) \psi_{0}\left(y^{\prime}\right)}{4 \pi r}+\int_{0}^{\infty} d m \psi_{m}(y) \psi_{m}\left(y^{\prime}\right) \frac{e^{-m r}}{4 \pi r}
\end{aligned}
$$


where $r=\left|\vec{x}-\vec{x}^{\prime}\right|$. As usual the potential is obtained upon integrating the Green's function times the source, Eq. (5), and we are interested in its form at the brane, i.e. $y=0$, namely,

$$
\begin{aligned}
\varphi(r, y=0) & =\int d^{3} x^{\prime} d y^{\prime} G\left(\vec{x}, y=0 ; \overrightarrow{x^{\prime}}, y^{\prime}\right) J_{\text {scalar }}\left(\overrightarrow{x^{\prime}}, y^{\prime} ; \overrightarrow{x_{0}}, y_{0}\right) \\
& =\frac{\lambda^{(p)} \psi_{0}(0) \psi_{0}\left(y_{0}\right)}{4 \pi r}+\lambda^{(p)} \int_{0}^{\infty} d m \psi_{m}(0) \psi_{m}\left(y_{0}\right) \frac{e^{-m r}}{4 \pi r},
\end{aligned}
$$

where now $r=\left|\vec{x}-\vec{x}_{0}\right|$, and (19) takes the asymptotic value

$$
\psi_{m}(0) \approx \frac{1}{\Gamma(\gamma-1)} \sqrt{\frac{m \epsilon}{2}}\left(\frac{m \epsilon}{2}\right)^{\gamma-2} .
$$

Finally, the potential becomes

$$
\varphi(r)=\frac{\lambda^{(p)}}{4 \pi r}\left(\frac{2+p}{2 \epsilon}\right)-\lambda^{(p)} \int_{0}^{\infty} d m \frac{1}{\Gamma(\gamma-1)}\left(\frac{m \epsilon}{2}\right)^{\gamma-1} e^{\gamma y_{0} / \epsilon} J_{\gamma}\left(m \epsilon e^{y_{0} / \epsilon}\right) \frac{e^{-m r}}{4 \pi r} .
$$

Next we further assume the source to be located at the brane, i.e. $y_{0}=0$. The explicit form of (24) now depends on whether the number of extra compact dimensions, $p$, is odd or even, and so we discuss each case separately.

\section{Odd number of extra compact dimensions}

In the case that $p$ takes odd values, $\gamma$ takes semi-integer values and is useful to use the relation

$$
m^{l+1 / 2} J_{l+1 / 2}(m \epsilon)=(-1)^{l} \sqrt{\frac{2}{\pi}} \epsilon^{l+1 / 2}\left(\frac{d}{\epsilon d \epsilon}\right)^{l} \frac{\sin (m \epsilon)}{\epsilon},
$$

in the integrand of (24). Upon evaluation of the integral we get

$$
\varphi(r)=\frac{\lambda^{(p)}}{4 \pi r} \frac{2+p}{2 \epsilon}-\frac{(-1)^{\gamma-\frac{1}{2}} \lambda^{(p)} \epsilon^{2 \gamma-1}}{2^{\gamma-3 / 2} \sqrt{\pi} \Gamma(\gamma-1)} \frac{1}{4 \pi r}\left(\frac{d}{\epsilon d \epsilon}\right)^{\gamma-\frac{1}{2}}\left(\frac{\pi}{2 \epsilon}-\frac{\arctan \left(\frac{r}{\epsilon}\right)}{\epsilon}\right) .
$$

Let us notice the first term of this expression is divergent at $r=0$. However such a term cancels out with the first term within parenthesis for every odd $p$. This leads to the form of the effective potential

$$
\varphi(r)=\frac{(-1)^{\gamma-\frac{1}{2}} \lambda^{(p)} \epsilon^{2 \gamma-1}}{2^{\gamma-3 / 2} \sqrt{\pi} \Gamma(\gamma-1)} \frac{1}{4 \pi r}\left(\frac{d}{\epsilon d \epsilon}\right)^{\gamma-\frac{1}{2}}\left(\frac{\arctan \left(\frac{r}{\epsilon}\right)}{\epsilon}\right)
$$

As an example, let us work out the case in which we have only one compact extra dimension, ie $p=1 \Rightarrow \gamma=5 / 2$. From (27) we obtain

$$
\varphi(r)=\frac{2 q_{s}^{(1)}}{3 \pi \epsilon}\left[3 \frac{\arctan \left(\frac{r}{\epsilon}\right)}{\frac{r}{\epsilon}}+\frac{5}{\left(1+\frac{r^{2}}{\epsilon^{2}}\right)}+2 \frac{\frac{r^{2}}{\epsilon^{2}}}{\left(1+\frac{r^{2}}{\epsilon^{2}}\right)^{2}}\right],
$$


where $q_{s}^{(1)}=\frac{3 \lambda^{(1)}}{8 \pi \epsilon}$. The finite value of the potential at the 3D point position of the source takes the value

$$
\lim _{r \rightarrow 0} \varphi(r)=\frac{16 q_{s}^{(1)}}{3 \pi \epsilon}
$$

evidently regularized by the existence of $\epsilon$ and $R$. Using (28) we can compute the effective self-energy of the point charge, as determined by a 3D observer

$$
E_{\text {self }}^{(p=1)}:=\frac{1}{2} \int_{\mathbb{R}^{3}} d^{3} x(\nabla \varphi)^{2}=\frac{85\left(q_{s}^{(1)}\right)^{2}}{9 \epsilon}
$$

\section{Even number of extra compact dimensions}

In the case that $p$ takes even values $\gamma$ is integer and we can use the relation

$$
m^{l} J_{l}(m \epsilon)=(-1)^{l} \epsilon^{l}\left(\frac{d}{\epsilon d \epsilon}\right)^{l-1}\left(-\frac{J_{1}(m \epsilon) m}{\epsilon}\right)
$$

in (24), to obtain [55]

$$
\varphi(r)=\frac{(-1)^{\gamma+1} \lambda^{(p)} \epsilon^{2 \gamma-1}}{2^{\gamma-1} \Gamma(\gamma-1)} \frac{1}{4 \pi r}\left(\frac{d}{\epsilon d \epsilon}\right)^{\gamma-1}\left(\frac{r}{\epsilon^{2} \sqrt{r^{2}+\epsilon^{2}}}\right) .
$$

As an example, let us consider the lowest even value for $p$ : $p=2 \Rightarrow \gamma=3$. From (32) we obtain

$$
\varphi(r)=\frac{q_{s}^{(2)}}{8 \epsilon}\left[\frac{8}{\sqrt{1+\frac{r^{2}}{\epsilon^{2}}}}+\frac{4}{\left(1+\frac{r^{2}}{\epsilon^{2}}\right)^{\frac{3}{2}}}+\frac{3}{\left(1+\frac{r^{2}}{\epsilon^{2}}\right)^{\frac{5}{2}}}\right]
$$

where $q_{s}^{(2)}=\frac{\lambda^{(2)}}{2 \pi \epsilon}$. In this case the finite value of the potential at the $3 \mathrm{D}$ position of the source is

$$
\lim _{r \rightarrow 0} \varphi(r)=\frac{15 q_{s}^{(2)}}{8 \epsilon}
$$

whereas the source self-energy is given by

$$
E_{\text {self }}^{(p=2)}=\frac{51975 \pi^{2}\left(q_{s}^{(2)}\right)^{2}}{65536 \epsilon}
$$

\section{ELECTROSTATIC POTENTIAL}

The procedure to compute this potential is similar to the one we used in the scalar case. In the spirit of avoiding repetition, we describe briefly the computation giving special emphasis 
to the aspects that are different with respect to the scalar case. A previous discussion of the photon Green's function analysis in the RSII- $p$ scenario can be found in [8]. We begin by considering the $(5+p) \mathrm{D}$ action

$$
S=\int d^{4} x \prod_{i=1}^{p} R_{i} d \theta_{i} d y \sqrt{|g|}\left(\frac{1}{4} g^{M N} g^{P Q} F_{M P} F_{N Q}+A_{M} J_{\text {gauge }}^{N}\right),
$$

leading to the equation of motion

$$
\frac{1}{\sqrt{|g|}} \partial_{M}\left(\sqrt{|g|} g^{M P} g^{N Q} F_{P Q}\right)=-j_{g a u g e}^{N} .
$$

We consider a static source along the brane, uniformly distributed along the $p$ extra compact dimensions, namely

$$
\sqrt{|g|} j_{\text {gauge }}^{N}=\rho^{(p)} \delta_{0}^{N} \delta^{3}\left(\vec{x}-\vec{x}_{0}\right) \delta\left(y-y_{0}\right)
$$

where $\rho^{(p)}$ is the charge density. Now we write down the equation of motion for the gauge field in the background (2). In order to do this, it is convenient to fix the gauge $A^{y}=0$ and $A^{\theta_{i}}=0$, which is consistent with the value $J^{\theta_{i}}=0$ for the components of the current density in the directions of the compact extra dimensions. Thus Eq. (37) becomes

$$
\mathcal{O} \hat{A}^{\sigma}-e^{-p|y| / \epsilon} \partial^{\sigma} \partial_{\mu} \hat{A}^{\mu}=-R^{-p} e^{p|y| / \epsilon} \sqrt{g} j^{\sigma}
$$

where we assume equal size compact dimensions, $R_{i}=R, i=1, \ldots, p$, and the differential operator $\mathcal{O}$ is defined as

$$
\mathcal{O}:=e^{-(p+2)|y| / \epsilon}\left(-\partial_{y}^{2}+\frac{p+2}{\epsilon} \operatorname{sgn}(y) \partial_{y}+e^{2|y| / \epsilon} \square\right),
$$

and $\hat{A}^{\nu}=\eta^{\nu \mu} A_{\mu}$. Inspection of equation (39) reveals the term $\partial_{\mu} \hat{A}^{\mu}$ is pure gauge on the brane, so we drop it from now on [8].

To solve (39) let us notice that the differential operator (40) is invariant under the change $y \rightarrow-y$, so the solutions will inherit such symmetry. This is important since we are looking for the potential on the brane. We shall adopt again the Green's function method. As in the scalar case, the necessary tools are the eigenfunctions and eigenvalues of the differential equation.

The eigenfunctions and eigenvalues for the $p$ compact modes are the same as those for the scalar field, Eq. (12). As for the noncompact modes depending upon $y$ and subject 
to the boundary conditions (13) they fulfill again a Bessel equation and have the following form

$$
\phi_{0}=\sqrt{\frac{p}{2 \epsilon}}, \quad \phi_{m}(y)=e^{\nu y / \epsilon} \sqrt{\frac{m \epsilon}{2}}\left[a_{m} J_{\nu}\left(m \epsilon e^{y / \epsilon}\right)+b_{m} N_{\nu}\left(m \epsilon e^{y / \epsilon}\right)\right],
$$

where

$$
\nu \equiv \frac{p+2}{2}
$$

and the constants $a_{m}, b_{m}$ are defined as in (17), with $\gamma$ replaced by $\nu$. The modes are normalized in the form $\int_{-\infty}^{\infty} d y e^{-p|y| / \epsilon} \phi_{0}^{2}=1$ and $\int_{-\infty}^{\infty} d y e^{-p|y| / \epsilon} \phi_{m}(m \epsilon) \phi_{m^{\prime}}\left(m^{\prime} \epsilon\right)=\delta\left(m-m^{\prime}\right)$. Formally the Green's function, its low energy approximation and the static potential on the brane are obtained from (18), (20) and (24), replacing the scalar modes $\psi$ by the gauge modes $\phi$ as well as the factor $\gamma$ by $\nu$. The electrostatic Green's function on the brane takes the form

$$
G_{\text {gauge }}\left(\vec{x}-\vec{x}^{\prime}, y=0, y^{\prime}\right)=\frac{p}{2 \epsilon} \frac{1}{4 \pi r}-\frac{1}{4 \pi r} \frac{e^{\nu y^{\prime} / \epsilon}}{\Gamma(\nu-1)}\left(\frac{\epsilon}{2}\right)^{\nu-1} \int_{0}^{\infty} d m m^{\nu-1} J_{\nu}\left(m y^{\prime}\right) e^{-m r} .
$$

Since we are interested in the potential for a source located on the brane, we have to evaluate the above expression in the limit $y^{\prime} \rightarrow 0$. As in the scalar case this limit is different depending on whether $p$ is either even or odd. They are given explicitly below.

\section{1. $\quad$ o odd}

In this case the potential gets the form

$$
A^{0}(r)=\frac{\sigma^{(5+p)}}{4 \pi R^{p} r} \sqrt{\frac{2}{\pi}} \frac{(-1)^{\nu} \epsilon^{2 \nu-1}}{\Gamma(\nu-1)(2)^{\nu-1}}\left(\frac{d}{\epsilon d \epsilon}\right)^{\nu-\frac{1}{2}}\left[\frac{\arctan \left(\frac{r}{\epsilon}\right)}{\epsilon}\right],
$$

where $r=\left|\vec{x}-\vec{x}_{0}\right|$. As an example notice that for one extra compact dimension $p=1$ one gets

$$
A^{0}(r)=\frac{2 e}{\epsilon \pi}\left(\frac{1}{1+\frac{r^{2}}{\epsilon^{2}}}+\frac{\arctan \left(\frac{r}{\epsilon}\right)}{\frac{r}{\epsilon}}\right), \quad e=\frac{e^{(6)}}{2 R \epsilon^{2}},
$$

which reduces to the Coulomb potential for $r \gg \epsilon$ and is finite at the 3D source position

$$
\lim _{r \rightarrow 0} A^{0}(r)=\frac{4 e}{\pi \epsilon}
$$

The self-energy in this case is

$$
E_{\text {self }}^{(p=1)}:=\frac{1}{2} \int_{\mathbb{R}^{3}} d^{3} x\left(\nabla A^{0}\right)^{2}=\frac{5 e^{2}}{32 \pi^{3} \epsilon}
$$


2. $p$ even

Now $\nu$ is an integer and

$$
A^{0}(r)=\frac{(-1)^{\nu} \sigma^{(5+p)} \epsilon^{2 \nu-1}}{2^{\nu-1} \Gamma(\nu-1) R^{p}} \frac{1}{4 \pi r}\left(\frac{d}{\epsilon d \epsilon}\right)^{\nu-1}\left(\frac{r}{\epsilon^{2} \sqrt{r^{2}+\epsilon^{2}}}\right) .
$$

Notice that for $p=2$,

$$
A^{0}(r)=\frac{e}{\epsilon}\left(\frac{1}{\sqrt{1+\frac{r^{2}}{\epsilon^{2}}}}+\frac{1}{2\left(1+\frac{r^{2}}{\epsilon^{2}}\right)^{\frac{3}{2}}}\right), \quad e=\frac{e^{(7)}}{R^{2} \epsilon},
$$

which becomes the Coulomb potential for $r \gg \epsilon$ and its finite at the 3D source position:

$$
\lim _{r \rightarrow 0} A^{0}(r)=\frac{3 e}{2 \epsilon}
$$

The source self-energy is now

$$
E_{\text {self }}^{(p=2)}=\frac{315 e^{2}}{16384 \pi \epsilon}
$$

The static potentials for $p=1, p=2$ and Coulomb's are compared in Fig. (2). Remarkably as we have mentioned, the electrostatic potentials corrected by the extra dimensions are finite at the 3D position of the charge.

It is interesting and natural to explore possible consequences of the modified electrostatic potentials we just obtained using known experiments like the Cavendish and scattering ones. We do so in the following section.

\section{PHENOMENOLOGY OF THE ELECTROSTATIC POTENTIAL}

\section{A. Cavendish experiment}

From the different results obtained to verify the accuracy of the electrostatic force, we have chosen the ones obtained by Plimpton and Lawton [43] and more recent modifications (see [44, 45] for a review of the different experiments). The reason is that this belongs to a series of experiments in which the main idea was to test the accuracy of Coulomb's force between charged particles using similar techniques as the one used by Cavendish to test the

gravitational force (see for instance [1] for a recent review on the different perspectives and experiments performed to test different aspects of electrodynamics). In the modern version 


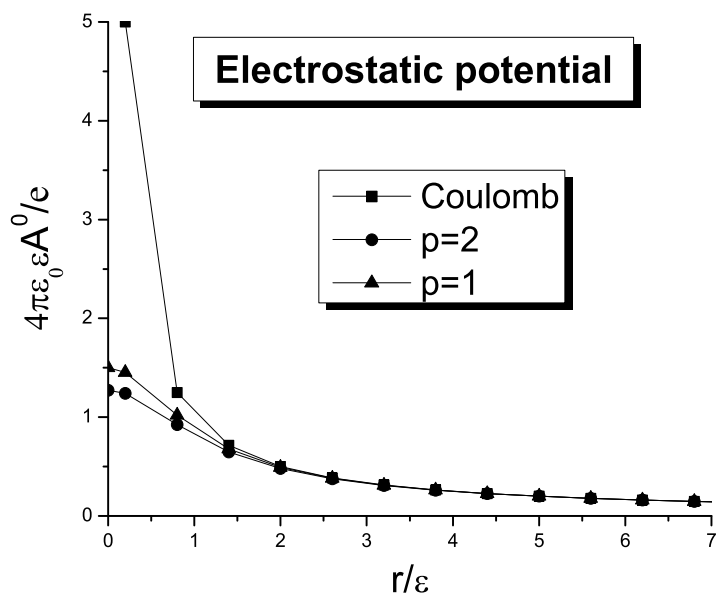

FIG. 2: Electrostatic potential of the point particle for the standard 4 D Coulomb case and $p=1,2$.

of Cavendish experiment we have a modified electromagnetic potential for a charge $Q$

$$
V=V_{C}+\delta V
$$

where $\delta V$ is the modification to the Coulomb potential. The idea behind the concentric charged spheres experiments is that only for the Coulomb potential the interior of a charged sphere is field free and therefore the potential there is a constant. Then the potential difference between a charged outer sphere and the uncharged inner sphere is zero only if the potential is that of Coulomb. Any deviation from this would imply a nonvanishing potential difference between the spheres that can be measured.

The potential of a sphere with a charge $Q$ and radius $c$ at a distance $r$ from the center is

$$
U(Q, r, c)=\frac{Q}{2 c r}[f(r+c)-f(|r-c|)]
$$

where the function $f$ is given by

$$
f(r)=\int_{0}^{r} d s s V(s, Q=1)
$$

It is easy to verify that for $V=\alpha / r, U(Q, r<c, c)=$ const., that is the potential is constant in the interior.

In the simplest version of the Cavendish experiment one has an outer sphere of radius $b$, charged to a certain voltage, and then measures the relative voltage difference to the 
uncharged inner sphere of radius $a<b$,

$$
\gamma_{a b}=\left|\frac{\mathcal{V}_{b}-\mathcal{V}_{a}}{\mathcal{V}_{b}}\right|=\left|\frac{U(Q, b, b)-U(Q, a, b)}{U(Q, b, b)}\right| .
$$

Plimpton and Lawton found that $\left|\gamma_{a b}\right| \leq 3 \times 10^{-10}$ with $a=0.696 \mathrm{~m}, b=0.762 \mathrm{~m}$. Here we will calculate $\left|\gamma_{a b}\right|$ for the two potentials corresponding to $p=1$ and $p=2$, namely

$$
\begin{aligned}
& v_{1}=\frac{2 Q}{\epsilon \pi}\left(\frac{1}{1+\frac{r^{2}}{\epsilon^{2}}}+\frac{\arctan \left(\frac{r}{\epsilon}\right)}{\frac{r}{\epsilon}}\right), \\
& v_{2}=\frac{Q}{\epsilon}\left(\frac{1}{\sqrt{1+\frac{r^{2}}{\epsilon^{2}}}}+\frac{1}{2\left(1+\frac{r^{2}}{\epsilon^{2}}\right)^{\frac{3}{2}}}\right) .
\end{aligned}
$$

The results, to first order in $\epsilon$ are

$$
\left|\gamma_{1 a b}\right|=\frac{\epsilon}{\pi b}, \quad\left|\gamma_{2 a b}\right|=\frac{\epsilon}{4 b}
$$

Taking into account the experimental bound of Plimpton and Lawton this means that $\epsilon \leq$ $7.18 \times 10^{-10} m$ or $\epsilon \leq 9.14 \times 10^{-10} m$ for $p=1$ and $p=2$, respectively.

The more recent version of the Cavendish experiment employs four concentric spheres of radii a, b, c, d in increasing order. The Outer sphere has a charge $Q$ and the next one $-Q$. Then the potential at radius $\mathrm{r}$ is given by

$$
U(Q, r, c, d)=\frac{Q}{2 d r}[f(r+d)-f(|r-d|)]-\frac{Q}{2 c r}[f(r+c)-f(|r-c|)],
$$

The experiment sets a bound for the ratio of the potential differences between the two uncharged spheres and the two outer spheres

$$
\gamma_{a b c d}=\left|\frac{\mathcal{V}_{b}-\mathcal{V}_{a}}{\mathcal{V}_{c}-\mathcal{V}_{d}}\right|=\left|\frac{U(Q, b, c, d)-U(Q, a, c, d)}{U(Q, c, c, d)-Q(Q, d, c, d)}\right| .
$$

Williams et al. [46] found that $\left|\gamma_{a b c d}\right| \leq 2 \times 10^{-16}$ with $a=0.60 \mathrm{~m}, b=0.94 \mathrm{~m}, c=0.947 \mathrm{~m}$ and $d=1.27 \mathrm{~m}$. Here we will calculate $\left|\gamma_{a b c d}\right|$ for the two potentials corresponding to $p=1$ and $p=2$, and using the experimental limits to constrain $\epsilon$. A straightforward calculation gives, to leading order in $\epsilon$

$$
\begin{aligned}
& \gamma_{1 a b c d}=\frac{c d\left(\frac{4(c-d)(c+d)\left(-2 a^{2}+c^{2}+d^{2}\right)}{\left(a^{2}-c^{2}\right)^{2}\left(a^{2}-d^{2}\right)^{2}}+\frac{4\left(2 b^{2}(c-d)(c+d)-c^{4}+d^{4}\right)}{\left(b^{2}-c^{2}\right)^{2}\left(b^{2}-d^{2}\right)^{2}}\right)}{3 \pi(c-d)} \epsilon^{3}+O\left(\epsilon^{4}\right),
\end{aligned}
$$

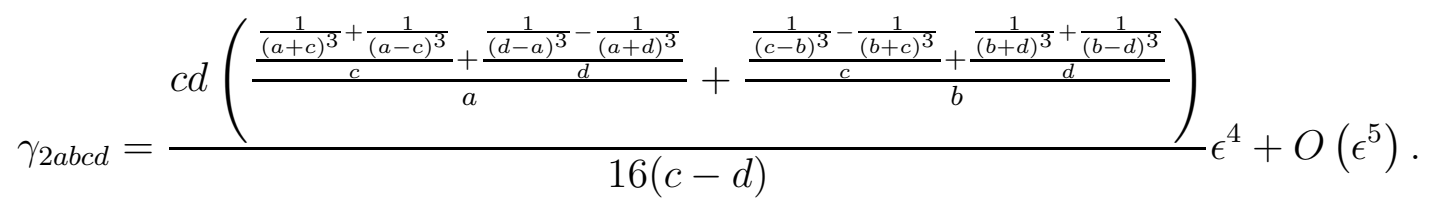


Taking into account the experimental value obtained by Williams et al. $\left(\left|\gamma_{a b c d}\right| \leq 2 \times 10^{-16}\right.$ ) the corresponding bounds for $\epsilon$ are $\epsilon \leq 2.80 \times 10^{-7} \mathrm{~m}$ or $\epsilon \leq 4.02 \times 10^{-6} \mathrm{~m}$ for $p=1$ and $p=2$, respectively. In this case the two sphere experiment gives a tighter constraint on $\epsilon$. The reason for this may be the peculiarities of the modification of the Coulomb potentials that in our case contains positive powers of $r$.

\section{B. Scattering by Helium atoms}

We shall study the collision of a particle of charge ze and mass $m$ with an atom of atomic number $Z$. Notice that an exact formulation of this problem requires the use of a manybody Hamiltonian which describes all the particles of the system, however we shall make the assumption that the complicated interaction of the incident particle with the constituents of the atom can be accounted for by an effective electrostatic potential $V(r)$ in which the incident particle travels.

It is physically reasonable that the electrostatic potential in which the incident particle travels is well approximated by

$$
V(\vec{r})=z e\left[Z e v_{1,2}(\vec{r})+e \int \rho\left(\vec{r}^{\prime}\right) v_{1,2}\left(\left|\vec{r}-\vec{r}^{\prime}\right|\right) d^{3} \vec{r}^{\prime}\right]
$$

were $\vec{r}$ is the position vector of the incident particle and $v_{1,2}(\vec{r})$ are given by (45) and (49). The first term is due to the field of the nucleus and the second term is the potential of the atomic electrons, described in terms of an effective electron density $\rho$. It is worth mentioning that in this description we are neglecting all effects of symmetry and spin. For neutral atoms, the density satisfies

$$
\int \rho(\vec{r}) d^{3} \vec{r}=Z
$$

When the incident particle carries sufficiently high energy, the scattering amplitudes can be easily evaluated by the Born approximation

$$
f(\theta)=-\frac{m}{2 \pi \hbar^{2}} \int e^{i \vec{q} \cdot \vec{r}} V(\vec{r}) d^{3} \vec{r}
$$

where $\vec{q}=\vec{k}_{0}-\vec{k}$, and $\vec{k}_{0}$ and $\vec{k}$ are the initial and final momentum, respectively. Since the scattering is elastic, $|\vec{k}|=\left|\vec{k}_{0}\right|=k$. Thus introducing Eq. (159) in (61) and making the 
following change of variable $\vec{R}=\vec{r}-\vec{r}^{\prime}$ we have

$$
\begin{aligned}
f_{1,2}(\theta) & =-\frac{m e^{2}}{2 \pi \hbar^{2}}\left[z Z \int e^{i \vec{q} \cdot \vec{r}} v_{1,2}(\vec{r}) d^{3} \vec{r}-z \int e^{i \vec{q} \cdot \vec{R}} v_{1,2}(\vec{R}) d^{3} \vec{R} \int \rho\left(\vec{r}^{\prime}\right) e^{i \vec{q} \cdot \vec{r}^{\prime}} d^{3} \vec{r}^{\prime}\right] \\
& =-\frac{m e^{2} z}{2 \pi \hbar^{2}}[Z-F(\vec{q})] \int e^{i \vec{q} \cdot \vec{r}} v_{1,2}(\vec{r}) d^{3} \vec{r}
\end{aligned}
$$

$F(\vec{q})$ is called the form factor of the atom. We defined $F(\vec{q})$ as

$$
F(\vec{q})=\int \rho(\vec{r}) e^{i \vec{q} \cdot \vec{r}} d^{3} \vec{r}
$$

When the potential is spherically symmetric, the angular integration can be performed to give

$$
f_{1,2}(\theta)=-\frac{2 m e^{2} z}{\hbar^{2}}[Z-F(\vec{q})] \int_{0}^{\infty} \frac{\sin (q r)}{q r} v_{1,2}(r) r^{2} d r
$$

with $q=|\vec{q}|=2 k \sin (\theta / 2)$ and $r=|\vec{r}|$. The evaluation of this integral depends on the form that $v_{1,2}(r)$ takes. We first calculate $f_{1}(\theta)$

$$
f_{1}(\theta)=-\frac{4 m e^{2} z}{\pi \hbar^{2}} \frac{1}{\epsilon q}[Z-F(\vec{q})] \int_{0}^{\infty} \sin (q r)\left(\frac{1}{1+\frac{r^{2}}{\epsilon^{2}}}+\frac{\arctan \left(\frac{r}{\epsilon}\right)}{\frac{r}{\epsilon}}\right) r d r
$$

using the following relations (see [47])

$$
\begin{aligned}
\int_{0}^{\infty} \frac{\sin (q r)}{1+\frac{r^{2}}{\epsilon^{2}}} r d r & =\frac{\pi}{2} \epsilon^{2} e^{-q \epsilon}, \\
\int_{0}^{\infty} \sin (q r) \frac{\arctan \left(\frac{r}{\epsilon}\right)}{r} r d r & =\frac{\pi}{2} \frac{e^{-q \epsilon}}{q}
\end{aligned}
$$

$f_{1}(\theta)$ can be written as

$$
f_{1}(\theta)=-\frac{2 m e^{2} z}{\hbar^{2}}[Z-F(\vec{q})]\left[\frac{1}{q^{2}}+\frac{\epsilon}{q}\right] e^{-q \epsilon} .
$$

Considering the form of $v_{2}(r), f_{2}(\theta)$ can be expressed as

$$
f_{2}(\theta)=-\frac{2 m e^{2} z}{\hbar^{2}} \frac{1}{\epsilon q}[Z-F(\vec{q})] \int_{0}^{\infty} \sin (q r)\left(\frac{1}{\sqrt{1+\frac{r^{2}}{\epsilon^{2}}}}+\frac{1}{2\left(1+\frac{r^{2}}{\epsilon^{2}}\right)^{\frac{3}{2}}}\right) r d r
$$

Now let us consider the integrals

$$
\begin{aligned}
& \int_{0}^{\infty} \frac{\sin (q r)}{\sqrt{1+\frac{r^{2}}{\epsilon^{2}}}} r d r=\epsilon^{2} K_{1}(q \epsilon) \\
& \int_{0}^{\infty} \frac{\sin (q r)}{\left(1+\frac{r^{2}}{\epsilon^{2}}\right)^{\frac{3}{2}}} r d r=\epsilon^{3} q K_{0}(q \epsilon)
\end{aligned}
$$


where $K_{0}(x)$ and $K_{1}(x)$ are Bessel functions of zeroth and first order, respectively. Thus $f_{2}(\theta)$ takes the form

$$
f_{2}(\theta)=-\frac{2 m e^{2} z}{\hbar^{2}}[Z-F(\vec{q})]\left[\frac{\epsilon}{q} K_{1}(q \epsilon)+\frac{\epsilon^{2}}{2} K_{0}(q \epsilon)\right] .
$$

For Helium we can calculate the electron density as

$$
\rho(r)=Z\left(\frac{b^{3}}{\pi a_{0}^{3}}\right) e^{\frac{-2 b r}{a_{0}}},
$$

with $b$ being the effective charge and having the value 1.69 for Helium while $a_{0}$ is the Bohr radius. The form factor becomes

$$
F(q)=\frac{Z}{\left(1+\frac{a_{0}^{2} q^{2}}{4 b^{2}}\right)^{2}}
$$

The differential scattering cross section for elastic processes thus become

$$
\begin{aligned}
& \left(\frac{d \sigma}{d \Omega}\right)^{(p=1)}=\left(\frac{2 z Z}{a_{0} q^{2}}\right)^{2}\left[1-\frac{1}{\left(1+\frac{a_{0}^{2} q^{2}}{4 b^{2}}\right)^{2}}\right]^{2}[1+q \epsilon]^{2} e^{-2 q \epsilon} \\
& \left(\frac{d \sigma}{d \Omega}\right)^{(p=2)}=\left(\frac{2 z Z}{a_{0} q^{2}}\right)^{2}\left[1-\frac{1}{\left(1+\frac{a_{0}^{2} q^{2}}{4 b^{2}}\right)^{2}}\right]^{2}\left[q \epsilon K_{1}(q \epsilon)+\frac{q^{2} \epsilon^{2}}{2} K_{0}(q \epsilon)\right]^{2}
\end{aligned}
$$

For incident electrons, we set $z=-1$ and $Z=2$ for the Helium atom. To complete the analysis we compare the theoretical results with the corresponding experimental ones. This comparison is made explicit in Figures (3) and (4). For both $p=1,2$ a best agreement is attained when $\epsilon \sim 10^{-10} \mathrm{~m}$.

\section{DISCUSSION}

The ever increasing accuracy with which electrodynamics has been tested naturally lends itself to consider it as a probe to set bounds for possible deviations coming from the existence of extra dimensions. Amongst different models the so called Randall-Sundrum ones including a single 3-brane and $p$ extra compact dimensions (RSII- $p$ ) have provided simple scenarios that yield effects well under control. Take for example the Casimir force [20, 21]: In a nutshell the field modes corresponding to the extra dimensions add up to modify the usual 


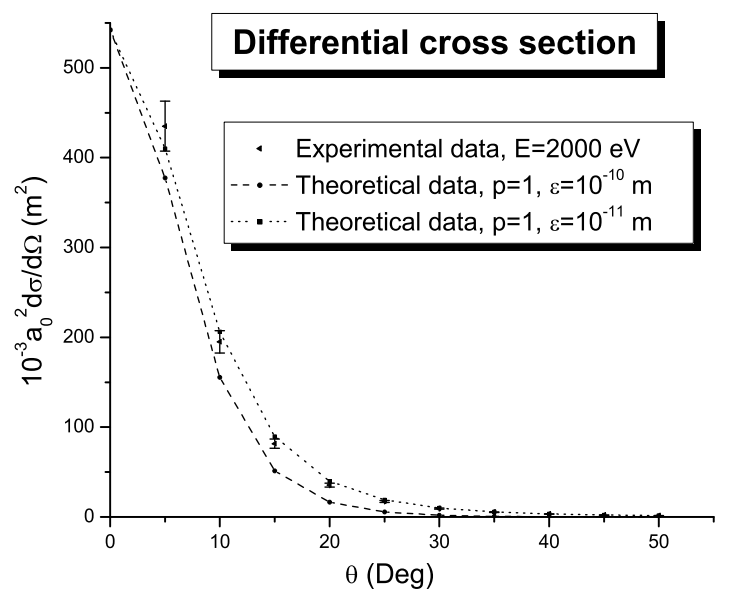

FIG. 3: Comparison of experimental differential cross section [48] with that corresponding to one compact dimension, Eq. (75).

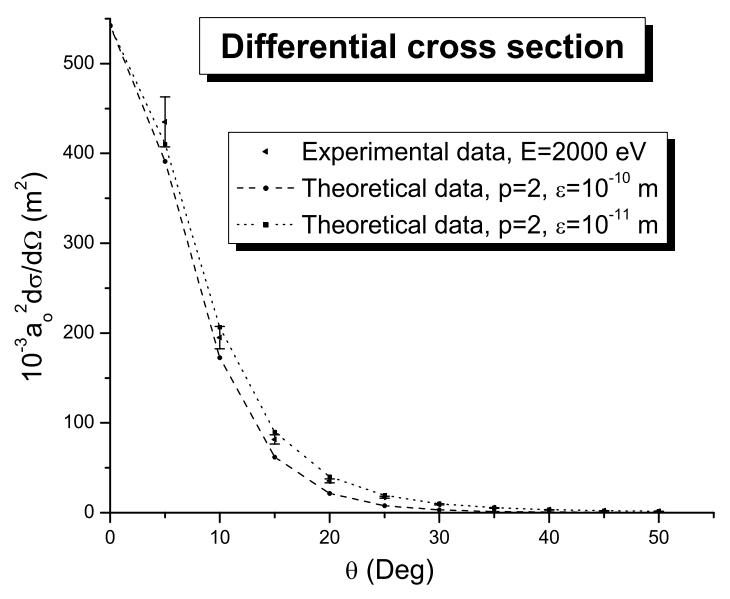

FIG. 4: Comparison of experimental differential cross section [48] with that corresponding to two compact dimensions, Eq. (76).

Casimir force expression and the deviations are assumed to be bounded by the uncertainties in the experimental data. This in turn sets bounds for the parameters of the brane model.

In this work we have explored the static potential produced by a scalar and a charged sources, respectively, in RSII- $p$. These sources are effectively pointlike from the perspective of an observer sitting in the usual 3D space. However they stretch uniformly along the $p$ compact dimensions thus having the structure of a $T^{p}$ torus. Remarkably the effective 
potentials turn out to be non-singular at the position in 3D space. At first one may think this is related to the fact the sources stretch along the extra dimensions, similarly as in models of charged spherical shells [49]. This is not the case as a more careful look reveals: the potential produced by either a charged ring or a torus is not finite at the source itself [50 52]. The RSII-p scenario thus allows to regularize the 3D potentials and selfenergies. Indeed the combined limit having AdS radius and compact size going to zero yields the usual standard divergent result.

We have determined the potentials in the low energy regime in terms of light modes; this entails approximating the continuous modes given in terms of Bessel functions by their small argument form whereas for the compact modes we keep the zero mode only. Within this approximation a delicate balance occurs between part of the massive sector contribution to the potential and the zero mode. Since the zero mode is responsible for the usual singular $1 / r$ term, the potential characteristic of massless fields, the balance just described regularizes such a divergence. Moreover the remaining effective potential becomes the usual $1 / r$ within a few times $\epsilon$ away from $r=0$ and provides finite selfenergies as determined from the usual $3 \mathrm{D}$ formulae.

To probe the effective potentials we proposed to consider two types of experiments. First we adopted the long known Cavendish experiment with two and four conducting spheres that is used to test the form of the Coulomb force. To be consistent with know experimental results for the case of two spheres a value of $\epsilon \sim 10^{-10} \mathrm{~m}$ is required. The four spheres setting however turns out to produce a milder bound $\epsilon \sim 10^{-7} \mathrm{~m}$, probably due to the positive powers of the correcting terms of the effective potentials when developing around $1 / r$. The second possibility we studied to test our effective potentials was to consider electrons scattered off by Helium. A comparison of the differential cross section modified by the RSII-p scenario with the curve fitting experimental data indicates consistency with a value of $\epsilon \sim 10^{-11} \mathrm{~m}$. In a previous work [6] we used the Lamb shift to set a bound of $\epsilon \sim 10^{-14} m$ for $p=1$, and $\epsilon \sim 10^{-13} \mathrm{~m}$, for $p=2$, which clearly are stronger than the ones obtained in the present work.

The fact that for both the scalar and electromagnetic case the potentials become well behaved leads naturally to the question of whether the same results holds for the gravitational case. This is work under study and will be reported elsewhere. Indeed, historically, finiteness of the potentials have led in the past to the idea that gravity regulates the self- 
energy of the charged point particle [53] as well as nonlinear field equations to achieve the finiteness of the electric field [54].

In the low energy regime we have focused on in this work there are some other possible directions which can be pursued. These include a reanalysis of the radiation reaction problem in both electromagnetic an gravitational cases as well as the complete understanding of the regularization of the potentials and in particular its relation to the topology of the sources together with their dimensionality.

\section{Acknowledgments}

HAMT acknowledges partial support from grant CONACyT-NSF Strong backreaction effects in quantum cosmology.

[1] A. S. Goldhaber and M. M. Nieto, Rev. Mod. Phys. 82, 939 (2010), 0809.1003.

[2] A. S. Goldhaber and M. M. Nieto, Rev. Mod. Phys. 43, 277 (1971).

[3] H. A. Lorentz, Teubner, Leipzig (1909), (orig. Leyden (1895)).

[4] M. Abraham, Ann. Phys. 10, 105 (1903).

[5] F. J. Dyson, Phys. Rev. 85, 631 (1952).

[6] H. A. Morales-Técotl, O. Pedraza, and L. O. Pimentel, Gen. Rel. Grav. 39, 1185 (2007), physics/0611241.

[7] S. L. Dubovsky, V. A. Rubakov, and P. G. Tinyakov, Phys. Rev. D62, 105011 (2000), hepth/0006046.

[8] S. L. Dubovsky, V. A. Rubakov, and P. G. Tinyakov, JHEP 08, 041 (2000), hep-ph/0007179.

[9] I. Oda, Phys. Lett. B496, 113 (2000), hep-th/0006203.

[10] L. Randall and R. Sundrum, Phys. Rev. Lett. 83, 4690 (1999), hep-th/9906064.

[11] L. Randall and R. Sundrum, Phys. Rev. Lett. 83, 3370 (1999), hep-ph/9905221.

[12] I. Antoniadis, N. Arkani-Hamed, S. Dimopoulos, and G. R. Dvali, Phys. Lett. B436, 257 (1998), hep-ph/9804398.

[13] N. Arkani-Hamed, S. Dimopoulos, and G. R. Dvali, Phys. Lett. B429, 263 (1998), hep$\mathrm{ph} / 9803315$. 
[14] I. Antoniadis, Phys. Lett. B246, 377 (1990).

[15] B. C. Allanach et al. (Beyond the Standard Model Working Group) (2004), hep-ph/0402295.

[16] C. Csaki (2004), hep-ph/0404096.

[17] R. Maartens, Living Rev. Rel. 7, 7 (2004), gr-qc/0312059.

[18] E. Elizalde, J. Phys. A39, 6299 (2006), hep-th/0607185.

[19] R. Maartens and K. Koyama (2010), 1004.3962.

[20] R. Linares, H. A. Morales-Técotl, and O. Pedraza, Phys. Rev. D81, 126013 (2010), 1003.4286.

[21] M. Frank, N. Saad, and I. Turan, Phys. Rev. D78, 055014 (2008), 0807.0443.

[22] T. Kaluza, Sitzungsber. Preuss. Akad. Wiss. Berlin (Math. Phys. ) 1921, 966 (1921).

[23] O. Klein, Z. Phys. 37, 895 (1926).

[24] M. J. Duff, B. E. W. Nilsson, and C. N. Pope, Phys. Rept. 130, 1 (1986).

[25] M. B. Green, J. Schwarz, and E. Witten (1987).

[26] O. Klein, Nature 118, 516 (1926).

[27] V. A. Rubakov, Phys. Usp. 44, 871 (2001), hep-ph/0104152.

[28] J. Scherk and J. H. Schwarz, Phys.Lett. B82, 60 (1979).

[29] J. Scherk and J. H. Schwarz, Nucl.Phys. B153, 61 (1979).

[30] M. Grana, Phys.Rept. 423, 91 (2006), hep-th/0509003.

[31] R. Blumenhagen, B. Kors, D. Lust, and S. Stieberger, Phys.Rept. 445, 1 (2007), hepth/0610327.

[32] M. R. Douglas and S. Kachru, Rev.Mod.Phys. 79, 733 (2007), hep-th/0610102.

[33] P. Candelas and S. Weinberg, Nucl.Phys. B237, 397 (1984).

[34] J. Garriga and T. Tanaka, Phys.Rev.Lett. 84, 2778 (2000), hep-th/9911055.

[35] M. Sasaki, T. Shiromizu, and K.-i. Maeda, Phys.Rev. D62, 024008 (2000), hep-th/9912233.

[36] W. D. Goldberger and M. B. Wise, Phys.Rev.Lett. 83, 4922 (1999), hep-ph/9907447.

[37] J. Lesgourgues and L. Sorbo, Phys.Rev. D69, 084010 (2004), hep-th/0310007.

[38] D. Maity, S. SenGupta, and S. Sur, Phys.Lett. B643, 348 (2006), hep-th/0604195.

[39] S. Das, A. Dey, and S. SenGupta, Europhys.Lett. 83, 51002 (2008), 0704.3119.

[40] A. Flachi, J. Garriga, O. Pujolas, and T. Tanaka, JHEP 0308, 053 (2003), hep-th/0302017.

[41] R. Linares, H. A. Morales-Técotl, and O. Pedraza, Phys. Rev. D77, 066012 (2008), arXiv:0712.3963 [hep-ph].

[42] R. Linares, H. A. Morales-Técotl, and O. Pedraza, Phys. Rev. D78, 066013 (2008), 0804.2042. 
[43] S. J. Plimpton and W. E. Lawton, Phys. Rev 50, 1066 (1936).

[44] L.-C. Tu, J. Luo, and G. T. Gillies, Rept. Prog. Phys. 68, 77 (2005).

[45] L.-C. Tu and J. Luo, Metrologia 41, S136 (2004).

[46] E. R. Williams, J. E. Faller, and H. A. Hill, Phys. Rev. Lett. 26, 721 (1971).

[47] I. Gradshteyn and I. Ryzhik, Table of Integrals, Series and Products (Academic Press Inc., 1980), ISBN 0122947606.

[48] R. H. J. Jansen, F. J. de Heer, H. J. Luyken, B. van Wingerden, and H. J. Blaauw, Journal of Physics B: Atomic and Molecular Physics 9, 185 (1976).

[49] F. Rohrlich, Classical Charged Particles (World Scientific, 2007), ISBN 9812700048.

[50] M. Andrews, Journal of Electrostatics 64, 664 (2006).

[51] B. P. Kondratev, A. S. Dubrovskii, N. G. Trubitsyna, and E. S. Mukhametshina, Technical Physics 53, 948 (2008).

[52] E. Y. Bannikova, V. G. Vakulik, and V. M. Shulga (2010), 1009.4324.

[53] R. L. Arnowitt, S. Deser, and C. W. Misner (1962), gr-qc/0405109.

[54] M. Born and L. Infeld, Proc. Roy. Soc. Lond. A144, 425 (1934).

[55] It is worth mentioning here an alternative approach to get the same results for both odd an even $p$. It amounts to using the completeness relation of the noncompact modes. In such a case the coefficient in front of $1 / r$ is proportional to the square of a Dirac delta and by using either dimensional regularization or distribution operations the coefficient vanishes thus obtaining the same result, ie that the potential is finite at the $3 \mathrm{D}$ position of the source [6]. 